\title{
Discriminating Among Alkali lons in Aqueous Solutions by Seebeck Voltage Measurement in Microfluidic Channels
}

\author{
Faramarz Hossein-Babaei, Benyamin Harkinezhad, Yasin Saedi \\ Electronic Materials Laboratory, Electrical Engineering Department, K. N. Toosi University of \\ Technology, Tehran, 16317-14191, Iran \\ fhbabaei@kntu.ac.ir
}

\begin{abstract}
:
Microfluidic systems allow fast and parallel analyses of fluid samples consuming microscopic quantities of the analytes. While varieties of information acquisition techniques from microchannels are practiced, novel methods for extracting information regarding the nature of the fluid analyte are sought after. Here, for the first time, we record the liquid Seebeck voltages established along the liquid-filled channels with predetermined temperature gradients, and correlate the results to the chemical composition of the analyte. Liquid Seebeck voltages are measured along microfluidic channels filled with aqueous solutions of $\mathrm{LiCl}, \mathrm{NaCl}$, and $\mathrm{KCl}$. At constant molality, these solutions demonstrate Seebeck coefficients of +250 , +350 , and $+500 \mu \mathrm{V} / \mathrm{K}$, respectively. It is shown that the information acquired can be used for differentiation between electrolytes of different compositions.
\end{abstract}

Keywords: Seebeck effect, bodily fluids, microfluidics, electrolytes, alkali ions

\section{Introduction}

Extracting analytical information from fluid analytes injected in microfluidic channels allows their detections and classifications important in numerous medical and industrial applications [1, 2]. Regardless of their different ionic mobilities, it is difficult to discriminate between alkali ions by conductometry in a channel as it varies with both concentration and nature of the unknown ion. Here, for the first time, we are presenting extraction of Seebeck coefficient data and show that they provide information uncorrelated with those related to conductometry regarding the nature of the electrolyte.

\section{Methods}

Structure of the designed microfluidic system is schematically presented in Fig. 1a-b. The system comprises a $1.2 \mathrm{~mm} \times 0.5 \mathrm{~mm} \times 2.5 \mathrm{~mm}$ channel terminated to two $70 \mu \mathrm{L}$ microreservoirs located at both ends. The system is

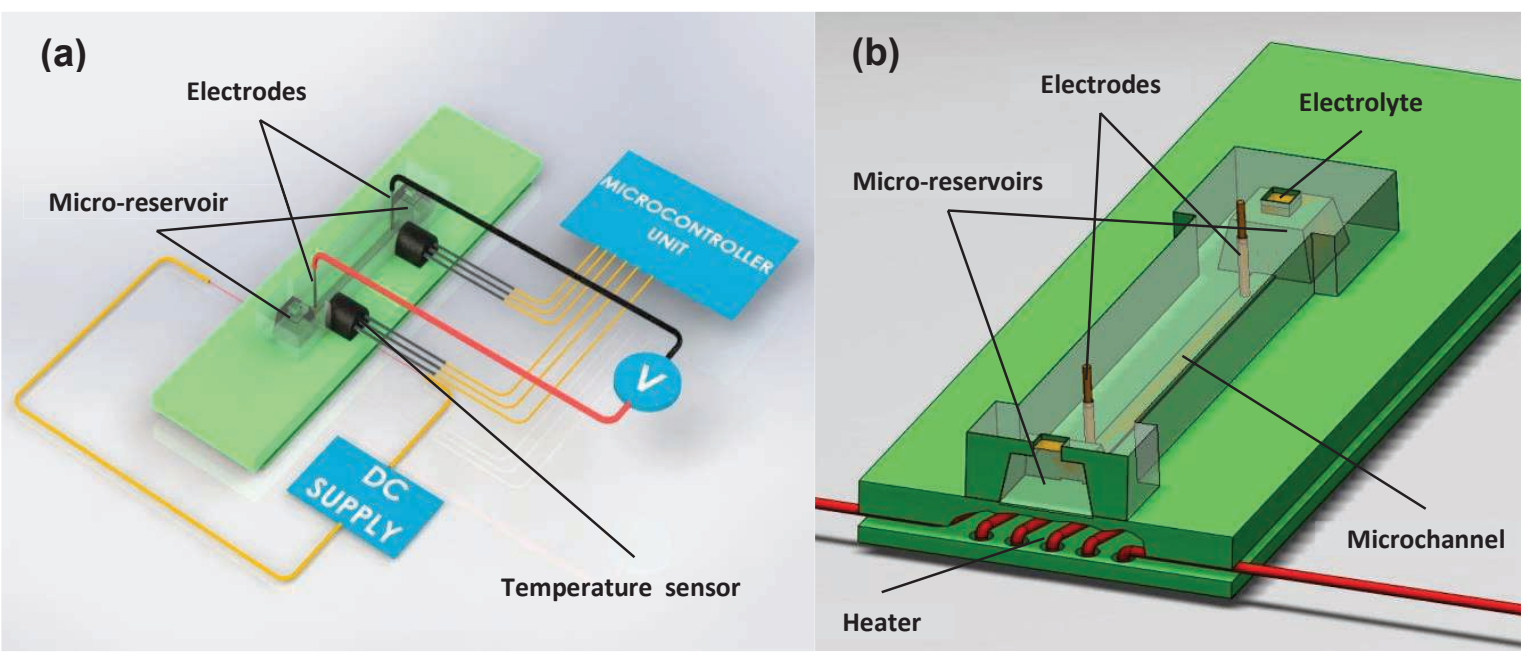

Fig. 1. Schematic diagram of the microfluidic system connected to the external measurement circuitry (a), and the magnified view of the hot reservoir and the heating microheater underneath (b). 
constructed on a PMMA chip by $\mathrm{CO}_{2}$ laser engraving; the fabrication method is described elsewhere [3].The temperature gradient along the channel is established by applying a controlled heating voltage to the microheater constructed underneath one of the microreservoirs.

Two independent temperature sensors are used to monitor the temperatures of the hot and cold ends of the channel. Two pure platinum wire segments, $0.35 \mathrm{~mm}$ in diameter, are laterally inserted into the channel via two holes devised close to the channel ends as depicted in Fig. 1.

Tested analytes are $\mathrm{LiCl}, \mathrm{NaCl}$, and $\mathrm{KCl} 0.1$ molar solutions in distilled water. The conductivity of each analyte is monitored using a standard conductometer prior to use; results are similar to the data available in the background literature. An analyte is introduced into the clod reservoir utilizing a sampler of $0.2 \mathrm{~mL}$ volume, which fills the channel and both reservoirs. Heating voltage is applied based on the prior experiences to establish the required temperature difference between the channel ends. Seebeck voltage established along the channel is measured between the electrodes after resuming temperature stability. The method used for Seebeck voltage measurement is as described in [4].

\section{Results}

The temperature of the hot end is repeatedly varied by applying voltage pulses of different amplitudes to the microheater. The temporal variations of the temperature difference between the channel ends and the established Seebeck voltage are presented in Fig. 2. Based on similar experiments, plots of Seebeck voltage vs. temperature difference are produced for different analytes; results are

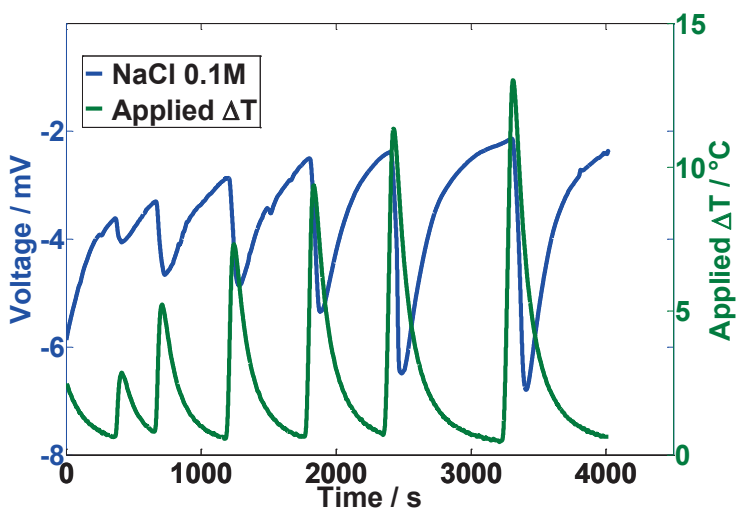

Fig.2. Raw data recorded for an analyte, showing the applied thermal gradient sequences and the resulting Seebeck voltage variations. given in Fig. 3. The discrimination between the electrolytes depicted in Fig. 3 is much more significant than differences observed by conductometry.

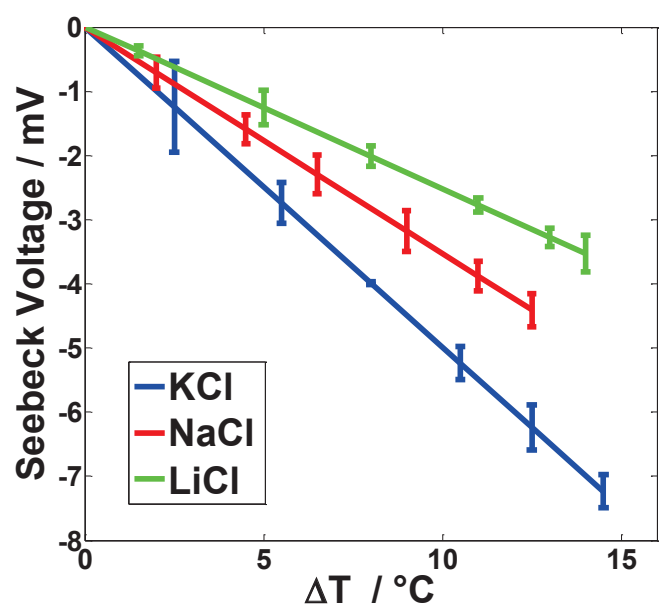

Fig. 3 Seebeck voltages of $0.1 \mathrm{~mol} . \mathrm{L}^{-1} \mathrm{LiCl}, \mathrm{NaCl}$ and $\mathrm{KCl}$ solutions vs. the temperature difference between the hot and the cold channel ends.

\section{Conclusion}

Based on the presented, and many other acquired experimental data, it is concluded that Seebeck voltage measurements along microfluidic channels provide useful information on the nature of the charged species present in the analyte inside the channel. The results provide information uncorrelated to the conductometry data; useful for analyte discrimination, e.g. $\mathrm{K}^{+}$and $\mathrm{Na}^{+}$ions in bodily fluids.

\section{References}

[1] Hossein-Babaei, Faramarz, and Ali Hooshyar Zare. "The selective flow of volatile organic compounds in conductive polymer-coated microchannels." Scientific Reports 7 (2017): 42299; DOI:10.1038/srep42299

[2] Hossein-Babaei, Faramarz, and Kianoosh Nemati. "A concept of microfluidic electronic tongue." Microfluidics and nanofluidics 13.2 (2012): 331-344. DOI: 10.1007/s10404-0120967-x

[3] Hossein-Babaei, Faramarz, Mohammad Paknahad, and Vahid Ghafarinia. "A miniature gas analyzer made by integrating a chemoresistor with a microchannel." Lab on a Chip 12.10 (2012): 1874-1880. DOI: 10.1039/C2LC00035K

[4] Hossein-Babaei, Faramarz, Saeed Masoumi, and Amirreza Noori. "Seebeck voltage measurement in undoped metal oxide semiconductors." Measurement Science and Technology 28.11 (2017): 115002. DOI: 10.1088/1361-6501/aa82a4 